\title{
Effect of phonon confinement on the dispersion relation and heat capacity in nanoscale Si membranes
}

\author{
J. Cuffe \\ Catalan Institute of \\ Nanotechnology, Campus UAB, \\ 08193 Bellaterra (Barcelona), \\ Spain \\ P-O. Chapuis \\ Catalan Institute of \\ Nanotechnology, Campus UAB, \\ 08193 Bellaterra (Barcelona), \\ Spain \\ Y. Pennec \\ Institut d'Electronique, de \\ Microélectronique et de \\ Nanotechnologie (IEMN), \\ Université de Lille 1, France \\ E. Chávez \\ Catalan Institute of \\ Nanotechnology, Campus UAB, \\ 08193 Bellaterra (Barcelona), \\ Spain \\ Dept. of Physics, Universitat \\ Autonoma de Barcelona, 08193 \\ Bellaterra (Barcelona), Spain

\begin{abstract}
E. H. El Boudouti Institut d'Electronique, de Microélectronique et de Nanotechnologie (IEMN), Université de Lille 1, France

LDOM, Faculté des Sciences, Morocco
\end{abstract} \\ Université Mohamed 1, Oujda, \\ B. Djafari-Rouhani \\ Institut d'Electronique, de \\ Microélectronique et de \\ Nanotechnologie (IEMN), \\ Université de Lille 1, France
}

\author{
A. Shchepetov \\ VTT Technical Research Centre \\ of Finland, PO Box 1000, 02044 \\ VTT, Espoo, Finland
}

\section{J. Ahopelto}

VTT Technical Research Centre of Finland, PO Box 1000, 02044 VTT, Espoo, Finland

\author{
C. M. Sotomayor Torres \\ Catalan Institute of Nanotechnology, Campus \\ UAB, 08193 Bellaterra (Barcelona), Spain \\ Dept. of Physics, Universitat Autonoma de \\ Barcelona, 08193 Bellaterra (Barcelona), Spain \\ Institució Catalana de Recerca i Estudis Avançats \\ (ICREA), 08010 Barcelona, Spain
}

*Email.. clivia.sotomayor@icn.cat

${ }^{\dagger}$ Present address: Centre de Thermique de Lyon (CETHIL) - CNRS - INSA Lyon, 9, rue de la Physique, Campus La Doua, 69621 Villeurbanne cedex, France

*Present address: Deutsche Forschungsgemeinschaft (DFG), Kennedyallee, 53175 Bonn, Germany. 


\section{ABSTRACT}

The effect of confinement on the acoustic phonon dispersion relation and heat capacity in free-standing silicon membranes is investigated, with thickness values down $\sim 8 \mathrm{~nm}$. The discrete phonon branches are observed by angle-resolved inelastic light scattering spectroscopy. The fundamental flexural mode was observed to have a scattering intensity nearly two orders of magnitude larger than the fundamental dilatational mode, which is ascribed to its large out-of-plane density of states and quadratic dispersion. The quadratic dispersion also results in a reduction of the phase and group velocities of the fundamental flexural mode by more than one order of magnitude compared to bulk values. To investigate the effect of this behavior on the thermal conductivity, we perform calculations based on continuum elasticity theory to estimate corresponding changes in the heat capacity. This work provides a basis to investigate the effects of the frequency and dimension dependence of other phonon properties, in particular in the sub$20 \mathrm{~nm}$ regime, where phonon-phonon relaxation times, density of states and thermal conductivity are expected to possess different spectral dependencies than for bulk materials.

\section{INTRODUCTION}

As channel widths of nanoelectronic devices scale well below $20 \mathrm{~nm}$, large power-densities and phonon-limited electron mobility are two of the greatest challenges towards increasing device performance. At the same time, great progress has been made in recent decades towards understanding and controlling the spectral distribution and propagation of phonons, for example, in optomechanical cavities [1] and phononic crystals [2,3]. These findings have given great impetus to phonon engineering as a strategy to deal with nanoscale thermal management for nanoelectronics and novel thermoelectric materials. Whereas previous discussions relating to phonons in thermal transport have emphasised "dominant" phonon wavelengths and mean free paths, there is a growing body of evidence that suggests that it is important to consider the entire phonon spectral distribution [4-7].

One well-known effect of decreasing dimensions on phonon transport is the limitation of the phonon mean free path [8] which results in a corresponding decrease in thermal conductivity. However, recent experimental work on periodically nanostructured silicon $[6,7,9]$ has suggested that the decrease in thermal conductivity observed is not due to this mean free path reduction alone, and further understanding of the effects of the non-linear phonon dispersion relation is sought to elucidate the physics behind thermal transport in nanostructures.

Nanomechanical resonators have also recently received much attention as ultrasensitive detectors of force [10], mass $[11,12]$, charge $[13,14]$ and spin [15], and as a platform for biosensing [16-18] and investigating quantum behaviour in extended objects $[19,20]$. Therefore, the understanding of the mechanical properties of these structures is of great importance. In the extreme sub- $10 \mathrm{~nm}$ regime, questions remain concerning the limits of validity of the continuum elasticity model and bulk elastic constants [21-23]. Non-contact experiments on structures in the nanoscale regime are an ideal method to investigate these fundamental questions.

The first direct studies of confined acoustic modes in ultrathin free-standing silicon membranes were performed with Raman spectroscopy [24,25]. Silicon membranes with thickness values of thirty nanometers were investigated with inelastic light scattering (ILS) spectroscopy, with the use of a triple-grating Raman spectrometer, with many discrete modes observed. However, in-plane phonon propagation of these acoustic modes was not examined.

In this work we investigate these issues further by measuring the changes in the acoustic dispersion in ultra-thin single-crystalline silicon membranes with thickness values down to $\sim 8 \mathrm{~nm}$. We then calculate the effect of this modification on the volumetric heat capacity for different phonon modes as a function of temperature.

\section{NOMENCLATURE}

$\begin{array}{ll}\text { A } n & \text { Flexural (Anti-Symmetric) mode of order } n \\ \text { S } n & \text { Dilatational (Symmetric) mode of order } n \\ \text { ILS } & \text { Inelastic Light Scattering } \\ \text { I } & \text { Scattering intensity } \\ \text { q } / / & \text { In-plane wavevector }\end{array}$

Free-standing single-crystalline silicon membranes are model systems for studies of phonon confinement, as they can be fabricated with precisely controlled dimensions and physical parameters, facilitating comparison with theoretical models. Moreover, being unsupported, a true, two-dimensional geometry is obtained and the analysis is free from any effects of a substrate.

The dispersion relations of the confined phonons were measured by angle-resolved Brillouin scattering spectroscopy. This technique has been shown to be an ideal method for characterising the acoustic properties of thin supported [26-28] and free-standing films [29-31] in a non-contact, nondestructive manner. The thickness values were obtained from reflectance measurements performed with a FilmTek 2000 spectroscopic reflectometer (Figure S1). The measurements were performed at four points around the membranes to reveal any thickness variation which was found to be less than 0.1 $0.5 \mathrm{~nm}$. The measured thickness values of the ultra-thin membranes investigated ranged from $7.8+/-0.1$ to $31.9+/-0.2$ $\mathrm{nm}$. The accuracy of the measurements is estimated to be better than one nanometer. For comparison, membranes with thickness values up to $400 \mathrm{~nm}$ were also investigated.

The measurements were performed in a backscattering configuration, with the incident wave vector, $\mathbf{k}$, making an 
angle $\theta$ to the surface normal of the sample as shown in Fig. 1(a). Due to the in-plane momentum conservation, light of freespace wavelength $\lambda$ is scattered inelastically by phonons with a in-plane wavevector component, $\mathrm{q}_{/ /}$given by:

$$
q_{/ /}=\frac{4 \pi}{\lambda} \sin \theta
$$

The dispersion relation $\omega\left(\mathrm{q}_{/ /}\right)$can then be measured by observing the spectral components of the inelastically scattered light as a function of the incident angle.

The spectral components of the inelastically scattered light were analyzed with a high resolution multipass $(3+3)$ Tandem Fabry-Perot Interferometer from JRS Scientific Instruments. The incident radiation was provided by a Diode Pumped Solid State Laser (DPSSL) from Oxxius, with a free-space wavelength, $\lambda$, of $532.6 \mathrm{~nm}$. This was focused on to the sample to a spot size of $12.5 \mu \mathrm{m}$ measured at Full-Width-Half-Max with an Olympus 10x microscope objective, with a numerical aperture of 0.5 . The measurements were performed at room temperature and the incident power was kept below a maximum of $5 \mathrm{~mW}$ for the $\sim 30 \mathrm{~nm}$ membranes, corresponding to a flux of less than $0.66 \mu \mathrm{W} / \mu \mathrm{m}^{2}$, and below a maximum of $1 \mathrm{~mW}$ for the $\sim 10 \mathrm{~nm}$ membranes corresponding to a flux of $0.125 \mu \mathrm{W} / \mu \mathrm{m}^{2}$, in order to avoid unwanted effects due to laser heating.

The acoustic dispersion relations in the membranes were calculated by solving the anisotropic acoustic wave equation

$$
\rho \frac{\partial^{2} U_{i}}{\partial t^{2}}=\frac{1}{2} \frac{\partial^{2}}{\partial x_{i}}\left[C_{i j k l}\left(\frac{\partial U_{k}}{\partial x_{l}}+\frac{\partial U_{l}}{\partial x_{k}}\right)\right]
$$

subject to stress-free boundary conditions on the membrane surfaces $\left(\sigma_{\mathrm{iz}}=0, i=x, y, z ; z=+/-d / 2\right)$, where $\rho$ is the mass density, $U$ is the acoustic displacement, and $C_{i j k l}$ is the elastic constant tensor. Solutions for the system were found using the partial wave method [32], and numerical solutions to the analytic equations were found using the procedure developed by Gazis et al. for surface acoustic waves on cubic crystals [33]. The elastic constants of Si used were $\mathrm{C}_{11}=165.7$ $\mathrm{GPa}, \mathrm{C}_{44}=63.9 \mathrm{GPa}$, and $\mathrm{C}_{12}=79.6 \mathrm{GPa}$ [34]. Anti-symmetric (A), symmetric (S) and shear horizontal (SH) solutions are found. The anti-symmetric and symmetric modes are also referred to as flexural and dilatational modes respectively, due to the nature of their characteristic displacements. Along highsymmetry directions, these modes can be considered as a coupling of the shear-vertical and longitudinal acoustic modes due to the boundaries of the membrane. The shear horizontal modes are uncoupled from the other modes along high symmetry directions.

To introduce the effects of confinement on the acoustic dispersion relation, we present ILS spectra for a $400 \mathrm{~nm}$ silicon membrane for incident angles between 15 and 60 degrees (Fig. 2(a)). Many spectral features are observed in comparison to the bulk case, for which only peaks corresponding to the longitudinal, transverse and surface acoustic waves can be seen [35]. The calculated dispersion relations of the flexural and dilatational modes in the [110] direction are shown in Fig. 2(b). From comparison with the calculated dispersion relation, the first two peaks nearest the central quasi-elastic peak are identified as the zero-order flexural and dilatational modes.

(a)

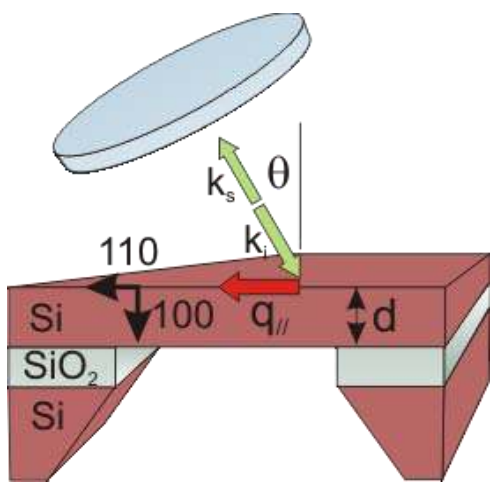

(b)

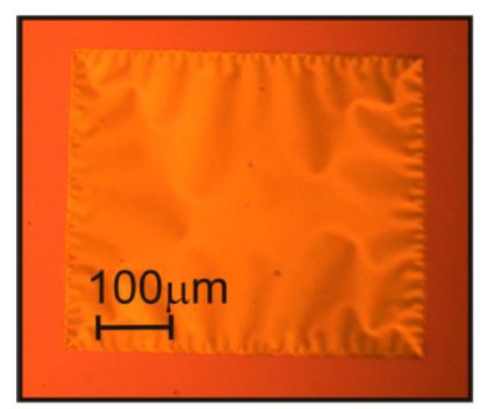

FIG. 1. (a) Scattering geometry and wavevector conservation. The measurements were performed in backscattering configuration, with a 10x Olympus microscope objective used both to focus the incident light and collect the inelastically scattered light. Wavevector conservation is also indicated as explained in the text. (b) Optical microscope image of the $30 \mathrm{~nm}$ Si membrane. The direction of the scattering wavevector, $q_{/ /}$, was kept in the [110] crystalline direction.

These are observed as independent peaks only at small wavevectors, and quickly become indistinguishable. The zeroorder flexural and dilatational modes may be thought of as interacting surface acoustic waves on either surface of the membrane. The flexural mode corresponds to when these modes are in-phase, resulting in a flexing of the membrane. The dilatational mode occurs when these waves are out-of-phase, thus resulting in a dilatational motion of the membrane. These modes can then be thought of as bonding and anti-bonding states of the surface acoustic modes, in analogy with bonding and anti-bonding states of molecular orbitals. The joining of these modes at large angles, or large values of $q / /$, is a consequence of the fact that at small wavelengths, the surface waves are confined to either surface and do not interact, thus 
behaving like a bulk surface acoustic waves. As the spectra were acquired in the [110] direction, these waves tend to the Pseudo Surface Acoustic (PSAW) wave velocity in silicon of $5085 \mathrm{~m} \mathrm{~s}^{-1}$.

At normal incidence, the modes that are observed at higher frequencies correspond to confined longitudinal and transverse standing waves, analogous to the confined modes in an openended pipe. The frequency separation between these modes for normal incidence is given by

$$
f_{L, T}=v_{L, T} / 2 d
$$

where $v_{L, T}$ represents the speed of sound of either longitudinal or transverse waves in the direction perpendicular to the membrane surface ([001] direction), and $d$ is the thickness of the membrane. At finite angles, these modes then acquire an in-plane component and exhibit dispersive behaviour. The frequency spacing between the higher-order modes scales inversely proportional to the thickness. Though all of the higher-order modes increase in frequency with a reducing value of the thickness, the zero-order flexural mode (A0) is the exception to this rule as it decreases in frequency for thinner membranes. This may be considered to be a representation of the fact that less energy is required to flex a thinner membrane.

(a)
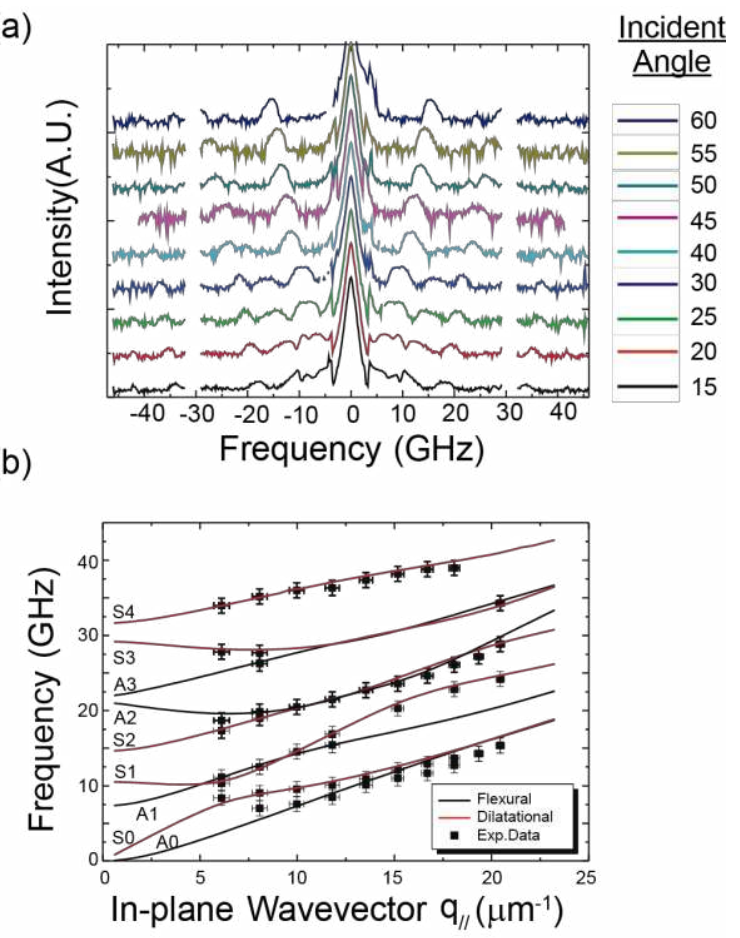

FIG. 2. (a) Typical Brillouin spectra acquired for a freestanding Si membrane with thickness of $400 \mathrm{~nm}$, as a function of the incident angle. A peak at $30.5 \mathrm{GHz}$ unrelated to the sample has been removed for clarity. (b) Calculated dispersion relation for flexural (black lines) and dilatational (red lines) modes, compared with experimental data (black squares).
Figure 3(a) shows high resolution spectra of the $30.7 \mathrm{~nm}$ thick silicon membrane. The peaks in the spectra are identified as the A0 modes of the membrane. The dispersion of this mode for ultra-thin membranes of various thickness values is shown in Fig. 3(b). The calculated dispersions, with no adjustable parameters, follow closely the experimental data. The most striking features are the quadratic dispersion and the reduction of phonon frequency of the flexural mode with decreasing thickness of the membranes: $\omega=A d q_{/ /}{ }^{2}$, where $A$ is a proportionality constant.

(a)

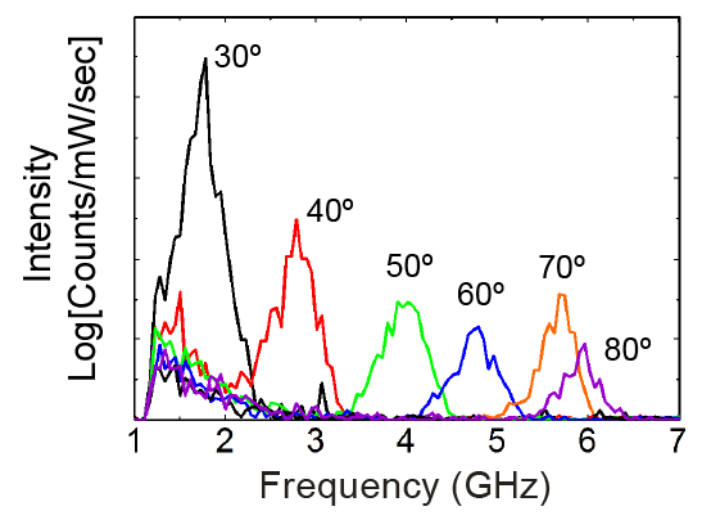

(b)

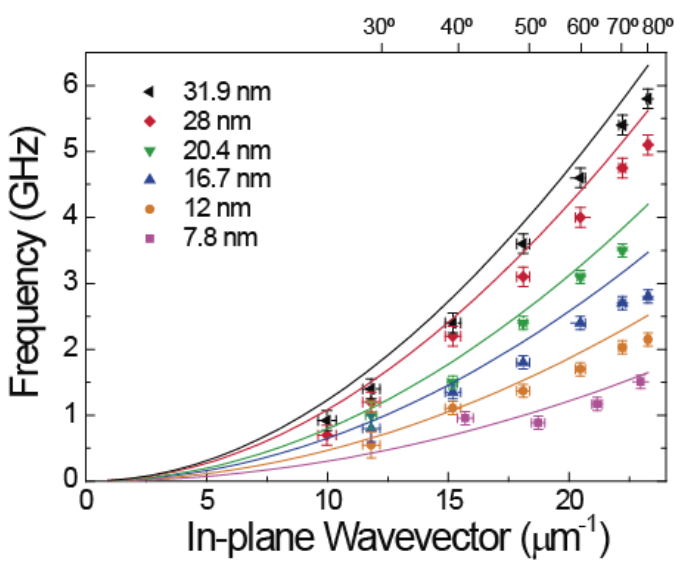

FIG. 3. (a) Spectra of the fundamental flexural (AO) mode of the $30.7 \mathrm{~nm}$ membrane for difference angles of incidence. The ILS spectra are shown for incident angles ranging from 30 to 80 degrees with respect to the sample normal as shown in Fig. 1(a). The measurements are performed with a resolution of $\sim 100 \mathrm{MHz}$. (b) Dispersion of the fundamental flexural modes (A0) in membranes with thickness values from 7.8 to $31.9 \mathrm{~nm}$. The dispersion relations are found to have a quadratic form, resulting in a larger density of states $D(\omega)$ with decreasing thickness. 
This quadratic dispersion results in a large density of states which, combined with the out-of-plane polarization, results in a large scattering amplitude [36]. To illustrate the relative magnitude of the out-of-plane density of states of this mode, a spectrum of the $30.7 \mathrm{~nm}$ membrane for a larger free spectral range is shown in Fig. 4, where both the fundamental flexural and dilatational modes are observed. The intensity of the peak assigned to the fundamental flexural (A0) mode is measured to be more than two orders of magnitude more intense than that of the fundamental dilatational (S0) mode. The quadratic dispersion relation also results in a reduction in phase velocity with thickness, $v_{p h}=A d q_{/ /}$, down to $300 \pm 40 \mathrm{~m} \mathrm{~s}^{-1}$ for the $7.8 \mathrm{~nm}$ membrane, which is commensurate with the reduction in the group velocity, $v_{g}=2 A d q_{/ /}$, down to $600 \pm 80 \mathrm{~m} \mathrm{~s}^{-1}$.

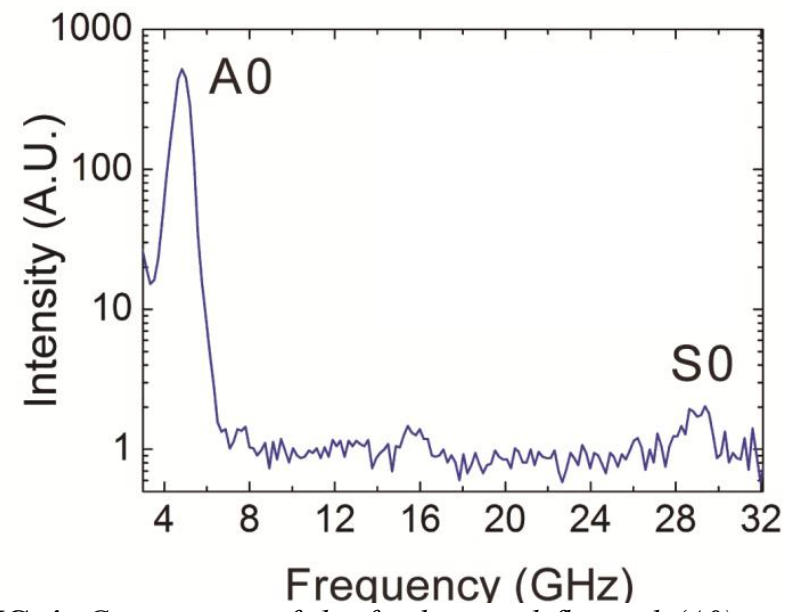

FIG 4. Comparison of the fundamental flexural (AO) and dilatational (SO) modes in the $30.7 \mathrm{~nm}$ thick membrane. The ILS spectrum was acquired at an incident angle of 70 degrees $\left(q_{/ /}=22 \mathrm{\mu m}^{-1}\right)$ with a resolution of $\sim 500 \mathrm{MHz}$. Note the intensity log scale.

A further consequence of the quadratic dispersion associated to the fundamental flexural mode is that the density of states $D(\omega) \propto 1 / d$ increases with decreasing membrane thickness, and thus more energy, $\int \hbar \omega D(\omega) f_{B E}(\omega, T) d \omega$, is stored in this mode, where $f_{B E}$ is the Bose-Einstein distribution function and $T$ the temperature. Therefore, the flexural mode could play a significant role in thermal transport in ultra-thin systems especially at low temperatures[37,38]. In the extreme case of graphene, this flexural mode was recently predicted to dominate the specific heat capacity and the lattice thermal conductivity[39].

To investigate this effect for ultra-thin $\mathrm{Si}$ membranes, we performed calculations of the volumetric heat capacity based on continuum elasticity theory. The volumetric heat capacity $C_{v}$ is defined as the amount of energy per unit volume to be supplied a system to increase its temperature by one degree Kelvin. The general form this quantity can be obtained by differentiation of the internal energy $(E)$ with respect to the temperature [40]

$$
C_{V}=\left.\frac{1}{V} \frac{\partial E}{\partial T}\right|_{V}=\frac{1}{V} \sum_{q, p} \hbar \omega_{q p} \frac{\partial n_{q p}}{\partial T}
$$

where $V$ is the volume and $n_{q p}$ is the Bose-Einstein equilibrium phonon distribution function. In the membrane system we cannot integrate in over all $q$-space, due to the discretization of the out-of-plane component of the wavevector. Therefore, the heat capacity is expressed as an integral in-plane with an out-of-plane summation:

$$
\begin{aligned}
C_{V} & =\frac{1}{S a} \sum_{n, p} \int_{0}^{q \max } \frac{\left(\hbar \omega_{q p}\right)^{2}}{K T^{2}} n_{q p}\left(n_{q p}+1\right) \frac{S}{(2 \pi)^{2}}(2 \pi) q_{/ /} d q_{/ /} \\
& =\frac{1}{2 \pi a} \sum_{n, p} \int_{0}^{q \max } c\left(\omega_{q p}\right) q_{/ /} d q_{/ /} ; \quad c\left(\omega_{q p}\right) \equiv \frac{1}{K_{B}}\left(\frac{\hbar \omega_{q p}}{T}\right)^{2} n_{q p}\left(n_{q p}+1\right)
\end{aligned}
$$

where $c\left(\omega_{q p}\right)$ is defined as the spectral density of the heat capacity, $S$ is the total surface area of the membrane and $d$ is the thickness and $q_{\max }$ is the maximum value of the in-plane wavevector limited by Debye cut-off [41]. Figure 5 shows that the temperature dependence of the specific heat in the lowtemperature regime $(\mathrm{T}<4 \mathrm{~K})$ departs from $\propto \mathrm{T}^{3}$ to $\propto \mathrm{T}$ behavior for 10 and $5 \mathrm{~nm}$ thick membranes. The explanation for this departure is that the flexural polarization gives the highest contribution to the total volumetric heat capacity, as shown in Fig. 5(b) for a $10 \mathrm{~nm}$ thick membrane, which reflects the fact that the dispersion is quadratic. The contribution of shear waves becomes the most important above $4 \mathrm{~K}$, with $38 \%$ of the total specific heat, increasing to a maximum of $43 \%$ at $30 \mathrm{~K}$. Above $\mathrm{T}>300 \mathrm{~K}$ we find a convergence of the contribution of all polarizations. Figure 5(a) shows that the specific heat of thin membranes in the low temperature regime increases significantly with reducing thickness. 
(a)

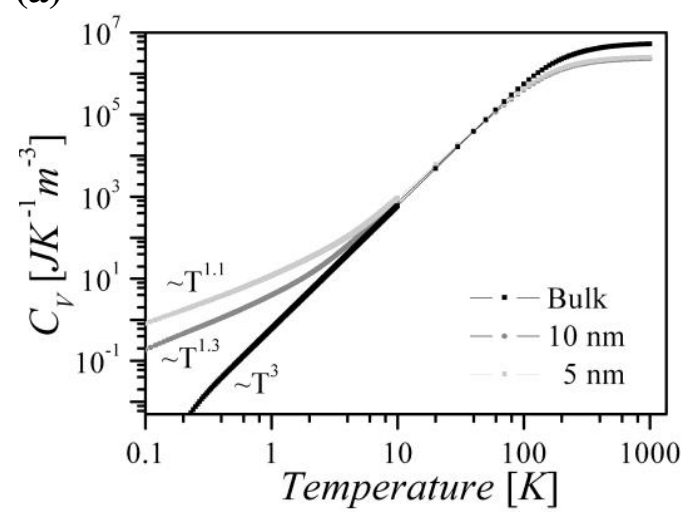

(b)

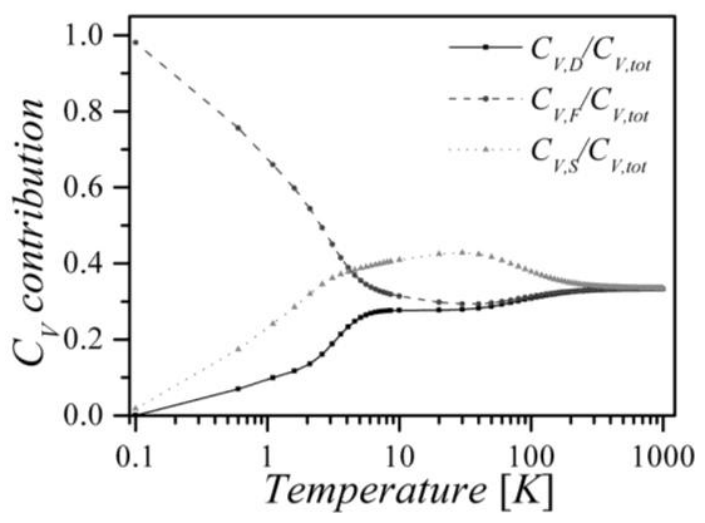

FIG. 5. (a) Specific heat as a function of temperature for bulk (black line), $10 \mathrm{~nm}$ (dark grey line) and $5 \mathrm{~nm}$ (grey line) thick membrane. (b) Contribution of each polarization to the total specific heat for $10 \mathrm{~nm}$ thick membrane. The black, dark grey dashed and grey dotted lines represent the contribution of dilatational $\left(C_{V, D}\right)$, flexural $\left(C_{V, F}\right)$ and shear $\left(C_{V, S}\right)$ waves respectively.

In summary, the in-plane propagation of confined acoustic modes in ultra-thin silicon membranes has been investigated, with measured thickness values down to $7.8 \mathrm{~nm}$, in agreement with a parameter-free elasticity model. The fundamental flexural mode, which exhibits an out-of-plane polarisation and quadratic dispersion, was observed to have a scattering intensity nearly two orders of magnitude larger than the fundamental dilatational mode, which exhibits primarily an inplane polarization and linear dispersion. We have shown that at low temperatures this modified phonon dispersion relation causes a significant deviation from bulk behaviour, due to the relatively high population of the quadratic flexural branch. At room temperature and above where most electronic devices operate, however, the volumetric heat capacity shows no significant departure from bulk behaviour, even for thin films down to $5 \mathrm{~nm}$. In addition to this effect, further work is required to calculate the effects of modified dispersion on phonon lifetimes and thermal transport, including both the effects of the group velocity and the modified three-phonon scattering processes, which may play an important role for both thermal and electrical transport in nanoscale devices at room temperature.

\section{ACKNOWLEDGMENTS}

The authors acknowledge the financial support from the EU FP7 projects TAILPHOX (grant nr. 233883), NANOPOWER (grant nr. 256959), NANOPACK (grant nr. 216176) and NANOFUNCTION (grant nr. 257375); the Spanish MICINN projects ACPHIN (FIS2009-10150) nanoTHERM (CSD2010-00044), and AGAUR 2009-SGR-150 and the Academy of Finland (grant nr. 252598). The 400-nmthick samples were fabricated using facilities from the "Integrated nano and microfabrication Clean Room" ICTS funded by MICINN. J.C. gratefully acknowledges a doctoral scholarship from the Irish Research Council for Science, Engineering and Technology (ICRSET) and E.C. gratefully acknowledges a Becas Chile 2010 CONICYT fellowship from Chilean government.

\section{REFERENCES}

[1] Eichenfield M., Chan J., Camacho R. M., Vahala K. J., and Painter O., 2009, "Optomechanical crystals," Nature, 462, pp. 78-82.

[2] Sigalas M. M., Kushwaha M. S., Economou E. N., Kafesaki M., Psarobas I. E., Steurer W., Eleftherios, and Economou N., 2005, "Classical vibrational modes in phononic lattices: theory and experiment," $\mathrm{Z}$. Kristallogr, 220(9-10), pp. 765-809.

Pennec Y., Rouhani B. D., El Boudouti E. H., Li C., Hassouani Y. E., Vasseur J. O., Papanikolaou N., Benchabane S., Laude V., and Martinez A., 2010, "Simultaneous existence of phononic and photonic band gaps in periodic crystal slabs," Opt. Express, 18 (13)(13), pp. 14301-14310.

[4] Henry A. S., and Chen G., 2008, "Spectral Phonon Transport Properties of Silicon Based on Molecular Dynamics Simulations and Lattice Dynamic," Journal of Computational and Theoretical Nanoscience, 5, pp. $1-12$.

[5] Balandin A. A., and Wang K. L., 1998, "Significant decrease of the lattice thermal conductivity due to phonon confinement in a free-standing semiconductor quantum well," Phys. Rev. B, 58(3), pp. 1544-1549. 
[6] Yu J.-K., Mitrovic S., Tham D., Varghese J., and Heath J. R., 2010, "Reduction of thermal conductivity in phononic nanomesh structures.," Nature nanotechnology, 5(10), pp. 718-21.

[7] Tang J., Wang H.-T., Lee D. H., Fardy M., Huo Z., Russell T. P., and Yang P., 2010, "Holey silicon as an efficient thermoelectric material.," Nano letters, 10(10), pp. $4279-83$.

[8] Liu W., and Asheghi M., 2006, “Thermal Conductivity Measurements of Ultra-Thin Single Crystal Silicon Layers," Journal of Heat Transfer, 128(1), pp. 75-83.

[9] Hopkins P. E., Reinke C. M., Su M. F., Olsson R. H., Shaner E. A., Leseman Z. C., Serrano J. R., Phinney L. M., and El-Kady I., 2011, "Reduction in the thermal conductivity of single crystalline silicon by phononic crystal patterning.," Nano letters, 11(1), pp. 107-12.

[10] Mamin H. J., and Rugar D., 2001, "Sub-attonewton force detection at millikelvin temperatures," Applied Physics Letters, 79(20), p. 3358.

[11] Chaste J., Eichler A., Moser J., Ceballos G., Rurali R., and Bachtold A., 2012, "A nanomechanical mass sensor with yoctogram resolution.," Nature nanotechnology, 7(5), pp. 301-304.

[12] Yang Y. T., Callegari C., Feng X. L., Ekinci K. L., and Roukes M. L., 2006, "Zeptogram-scale nanomechanical mass sensing.," Nano letters, 6(4), pp. 583-6.

[13] Lassagne B., Tarakanov Y., Kinaret J., Garcia-Sanchez D., Garcia-Sanchez D., and Bachtold A., 2009, "Coupling mechanics to charge transport in carbon nanotube mechanical resonators.," Science (New York, N.Y.), 325(5944), pp. 1107-10.

[14] Steele G. A., Hüttel A. K., Witkamp B., Poot M., Meerwaldt H. B., Kouwenhoven L. P., and van der Zant H. S. J., 2009, "Strong coupling between singleelectron tunneling and nanomechanical motion.," Science (New York, N.Y.), 325(5944), pp. 1103-7.

[15] Rugar D., Budakian R., Mamin H. J., and Chui B. W., 2004, "Single spin detection by magnetic resonance force microscopy.," Nature, 430(6997), pp. 329-32.

[16] Jensen K., Kim K., and Zettl A., 2008, “An atomicresolution nanomechanical mass sensor.," Nature nanotechnology, 3(9), pp. 533-7.
[17] Burg T. P., Godin M., Knudsen S. M., Shen W., Carlson G., Foster J. S., Babcock K., and Manalis S. R., 2007, "Weighing of biomolecules, single cells and single nanoparticles in fluid.," Nature, 446(7139), pp. 1066-9.

[18] Arlett J. L., Myers E. B., and Roukes M. L., 2011, "Comparative advantages of mechanical biosensors.," Nature nanotechnology, 6(4), pp. 203-15.

[19] O'Connell A. D., Hofheinz M., Ansmann M., Bialczak R. C., Lenander M., Lucero E., Neeley M., Sank D., Wang H., Weides M., Wenner J., Martinis J. M., and Cleland A. N., 2010, "Quantum ground state and single-phonon control of a mechanical resonator.," Nature, 464(7289), pp. 697-703.

[20] Naik A., Buu O., LaHaye M. D., Armour A. D., Clerk A. A., Blencowe M. P., and Schwab K. C., 2006, "Cooling a nanomechanical resonator with quantum back-action.," Nature, 443(7108), pp. 193-6.

[21] Bernal R. A., Agrawal R., Peng B., Bertness K. A., Sanford N. A., Davydov A. V., and Espinosa H. D., 2011, "Effect of growth orientation and diameter on the elasticity of GaN nanowires. A combined in situ TEM and atomistic modeling investigation.," Nano letters, 11(2), pp. 548-55.

[22] Juvé V., Crut A., Maioli P., Pellarin M., Broyer M., Del Fatti N., and Vallée F., 2010, "Probing elasticity at the nanoscale: Terahertz acoustic vibration of small metal nanoparticles.," Nano letters, 10(5), pp. 1853-8.

[23] Gordon M. J., Baron T., Dhalluin F., Gentile P., and Ferret P., 2009, "Size effects in mechanical deformation and fracture of cantilevered silicon nanowires.," Nano letters, 9(2), pp. 525-9.

[24] Sotomayor Torres C. M., Zwick A., Poinsotte F., Groenen J., Prunnila M., Ahopelto J., Mlayah A., and Paillard V., 2004, "Observations of confined acoustic phonons in silicon membranes," Physica Status Solidi (c), 1(11), pp. 2609-2612.

[25] Groenen J., Poinsotte F., Zwick A., Sotomayor Torres C. M., Prunnila M., and Ahopelto J., 2008, "Inelastic light scattering by longitudinal acoustic phonons in thin silicon layers: From membranes to silicon-on-insulator structures," Phys. Rev. B, 77(4), p. 45420. 
[26] Zhang X., Bandhu R. S., Sooryakumar R., and Jonker B. T., 2003, "High-frequency standing longitudinal acoustic resonances in supported thin films," Phys. Rev. B, 67(7), p. 75407.

[27] Chirita M., Sooryakumar R., Xia H., Monteiro O. R., and Brown I. G., 1999, "Observation of guided longitudinal acoustic modes in hard supported layers," Phys. Rev. B, 60(8), p. R5153--R5156.

[28] Ghislotti G., Gagliardi A., Bottani C. E., Bertoni S., Cerofolini G. F., and Meda L., 1996, "Characterization of SOI-SIMOX structures using Brillouin light scattering," Materials Science and Engineering B, 36(13), pp. 129-132.

[29] Zhang X., Sooryakumar R., and Bussmann K., 2003, "Confinement and transverse standing acoustic resonances in free-standing membranes," Physical Review B, 68(11), pp. 1-5.

[30] Grimsditch M., Bhadra R., and Schuller I. K., 1987, "Lamb waves in unsupported thin films: A Brillouinscattering study," Phys. Rev. Lett., 58(12), pp. 12161219.

[31] Bhadra R., Grimsditch M., Schuller I. K., and Nizzoli F., 1989, "Brillouin scattering from unsupported Al films," Phys. Rev. B, 39(17), pp. 12456-12459.

[32] Solie L. P., and Auld B. A., 1973, "Elastic waves in free anisotropic plates," The Journal of the Acoustical Society of America, 54(1), pp. 50-65.

[33] Gazis D. C., Herman R., and Wallis R. F., 1960, "Surface Elastic Waves in Cubic Crystals," Phys. Rev., 119(2), pp. 533-544.
[34] McSkimin H. J., and Andreatch P., 1964, "Elastic Moduli of Silicon vs Hydrostatic Pressure at $25.0^{\circ} \mathrm{C}$ and $-195.8^{\circ} \mathrm{C}$," Journal of Applied Physics, 35(7), p. 2161.

[35] Stoddart P. R. R., Comins J. D. D., and Every a. G. G., 1996, "High-temperature studies of surface acoustic wave velocities in silicon by Brillouin scattering," Physica B: Condensed Matter, 219-220, pp. 717-719.

[36] Loudon R., and Sandercock J. R., 1980, “Analysis of the light-scattering cross section for surface ripples on solids," Journal of Physics C: Solid State Physics, 13(13), p. 2609.

Kühn T., and Maasilta I. J., 2007, “Maximizing phonon thermal conductance for ballistic membranes," Journal of Physics: Conference Series, 92(1), p. 012082.

[38] Schwab K. C., Henriksen E., Worlock J., and Roukes M., 2000, "Measurement of the quantum of thermal conductance," Nature, 404(6781), pp. 974-7.

[39] Lindsay L., Broido D. A., and Mingo N., 2010, "Flexural phonons and thermal transport in graphene," Phys. Rev. B, 82(11), p. 115427.

[40] Srivastava G. P., 1990, The Physics of Phonons, Taylor \& Francis.

[41] Huang M.-J., Chang T.-M., Chong W.-Y., Liu C.-K., and Yu C.-K., 2007, "A new lattice thermal conductivity model of a thin-film semiconductor," International Journal of Heat and Mass Transfer, 50(12), pp. 67-74. 